\title{
Study the atomic properties of 2 s shell for some atoms
}

\author{
Shaymaa A. Kadhim \\ Department of Physics, College of Science, Kufa University \\ E-mail: Shaimanuclear@yahoo.com
}

\begin{abstract}
Ground state energies and other properties of $2 \mathrm{~S}$ shell for some atoms as $\mathrm{Be}(\mathrm{Z}=4), \mathrm{B}(\mathrm{Z}=5), \mathrm{C}(\mathrm{Z}=6)$ and $\mathrm{N}(\mathrm{Z}=7)$ were calculated by using Hartree-Fock wave function. We found the values of potential energies in hartree unit $(3.8369,6.78565,10.18852$ and 14.41089) respectively and the other proprieties like expectation values of the position $\left\langle\mathrm{r}_{1}{ }^{\mathrm{m}}>\right.$ were in agreement with the published results. All the studied atomic properties were normalized.
\end{abstract}

Key words Atomic Hartree-Fock, atomic properties.

Article info.

Received: Apr. 2013

Accepted: Nov. 2013

Published: Dec. 2013
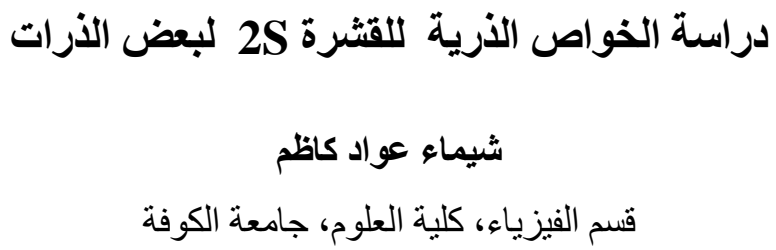

الخلاصة

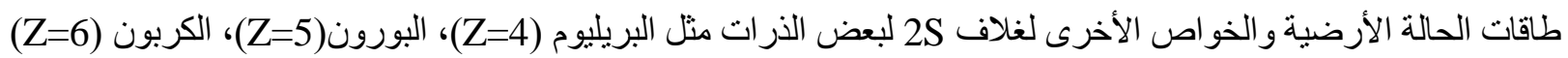

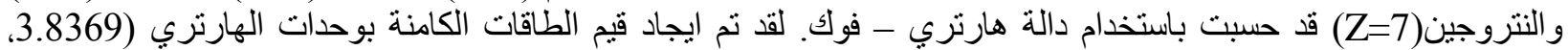

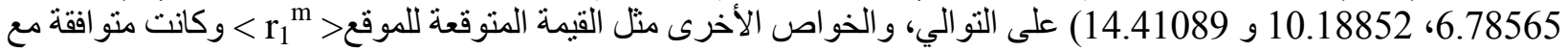

$$
\text { النتائج المنشورة عالميا. كل الخو اص الذرية المدروسة كانت عباريه. }
$$

\section{Introduction}

Any system can be described by a wave function; it is a mathematical expression which used to describe the system properties such as the position, momentum and energy etc. The exact calculation of wave functions for many electron atoms becomes difficult because of the many electron-electron repulsions that should be ignored for simplicity. There are two main kinds of the wave functions that have been used in the approximation methods, the first one is the uncorrelated wave function Hartree-Fock (HF) which used in the present research, and the second one is the correlated wave function such as Configuration Interaction (CI) [1].

\section{The uncorrelated wave function}

According to Pauli principle the wave function for system of electrons must change sign when the coordinates of any two electrons are interchanged, so that, from mathematical point of view, the many body 
wave function is written as Slater determinant [2]:

$\psi_{H F}(123 \ldots N)=\frac{1}{\sqrt{N !}}\left|\begin{array}{ccc}\Phi_{1}(1) & \cdots & \Phi_{1}(N) \\ \vdots & \ddots & \vdots \\ \Phi_{N}(1) & \cdots & \Phi_{N}(N)\end{array}\right|$

where $\frac{1}{\sqrt{N !}}$ the multiplying factor introduced to ensure that the wave function is normalized after integrating over all the space and spin coordinates such that.[3]

$$
\int \Psi^{*} \Psi d \tau=1
$$

where $\psi^{*}$ represents the complex conjugate of the wave function and the component of one particle function is referred to as spinorbital. Any spin-orbital may be written as the product of a space function and a spin function, where $\alpha$ - spin up and $\beta$-spin down.The spin wave functions are orthonormal, where:

$\int \alpha^{*}(1) \alpha(1) d \sigma_{1}=1$

$\int \beta^{*}(1) \beta(1) d \sigma_{1}=1$

$\int \alpha^{*}(1) \beta(1) d \sigma_{1}=0$

The wave function for $\mathrm{N}$-electron system is constructed from a basis set containing several orbital; this wave function can be written as [3].

$$
\begin{aligned}
& \Psi_{H F}(123 \cdots N)=\frac{1}{\sqrt{N !}}\left|\phi_{1}(1) \cdots \Phi_{N}(N)\right| \\
& \Phi_{i}(i)=\Phi(r, \theta, \Phi) \sigma(s) \\
& \Phi_{n l}=\sum_{i=1}^{j} c_{n}^{i} \chi_{n l m}
\end{aligned}
$$

where $\Phi$ is the spatial part, $\sigma(s)$ the spin part and the numbers in the parentheses denote the particle, and the subscripts $1,2 \ldots \mathrm{N}$ denotes the eigen state. The special function is defined by [1].

$$
\chi_{n l m}(r, \theta, \Phi)=R_{N L}(r) Y_{l m}(\theta, \Phi)
$$

the radial part of special part of the total wave function is given by:

$$
R_{n l}(r)=N_{n l m} S_{n l}(r)
$$

where $N_{n l m}$ is the normalization constant, and given by

$$
N_{n l m}=\sqrt{\frac{(2 \xi)^{2 n+1}}{(2 n) !}}
$$

and $S_{n l}(r)=r^{n-1} e^{-\xi r}$

where $S_{n l}(r)$ is called the Slater-TypeOrbitals (STO) and $\xi$ represents the orbital exponent.

\section{Results and discussion}

In this research we using the atomic data for Hartree-Fock wave function of Clementi and Roetti [4].

Table 1: Atomic data for Hartree-Fock functions for studied atoms [4].

\begin{tabular}{|c|c|c|c|c|c|c|c|c|c|c|c|}
\hline \multicolumn{3}{|c|}{$B e(\mathrm{Z}=4)$} & \multicolumn{3}{c|}{$B(\mathrm{Z}=5)$} & \multicolumn{3}{c|}{$C(\mathrm{Z}=6)$} & \multicolumn{3}{c|}{$N(\mathrm{Z}=7)$} \\
\hline$n$ & $\xi$ & $C_{2 s}$ & $n$ & $\xi$ & $C_{2 s}$ & $n$ & $\xi$ & $C_{2 s}$ & $n$ & $\xi$ & $C_{2 s}$ \\
\hline 1 & 3.47116 & -0.17092 & 1 & 4.44561 & -0.19484 & 1 & 5.43599 & -0.20814 & 1 & 6.45739 & -0.21677 \\
\hline 1 & 6.36861 & -0.01455 & 1 & 7.91796 & -0.01254 & 1 & 9.48256 & -0.01071 & 1 & 11.17200 & -0.00846 \\
\hline 2 & 0.77820 & 0.21186 & 2 & 0.86709 & 0.06941 & 2 & 1.05749 & 0.08099 & 2 & 1.36405 & 0.17991 \\
\hline 2 & 0.94067 & 0.62499 & 2 & 1.21924 & 0.75234 & 2 & 1.52427 & 0.75045 & 2 & 1.89734 & 0.67416 \\
\hline 2 & 1.48725 & 0.26662 & 2 & 2.07264 & 0.31856 & 2 & 2.68435 & 0.33549 & 2 & 3.25291 & 0.31297 \\
\hline 2 & 2.71830 & -0.09919 & 2 & 3.44332 & -0.12642 & 2 & 4.20096 & -0.14765 & 2 & 5.08238 & -0.14497 \\
\hline
\end{tabular}




\section{One- particle radial density distribution function $\mathrm{D}(\mathrm{r} 1)$}

We calculated one-electron radial density distribution function $D\left(r_{1}\right)$ (the probability of finding the electron in a spherical shell of distance $r_{1}$ from the nucleus) using the following equation [5] :

$$
D\left(r_{1}\right)_{2 s}=R_{2 s}^{2}\left(r_{1}\right) r_{1}^{2}
$$

where $R_{2 S}\left(r_{1}\right)$ represented the radial part of the wave function.

In Table 2 we listed the results of maximum values of $\mathrm{D}\left(\mathrm{r}_{1}\right)$ with the corresponding position $r_{1}$, Fig. 1 represents relation between $\mathrm{D}\left(\mathrm{r}_{1}\right)$ with the position for $2 \mathrm{~S}$ shell of studied atoms. We noted from Table 2 that the maximum values for $\mathrm{D}\left(\mathrm{r}_{1}\right)$ increase when the atomic number increases, that means the probability of finding an electron increase, and the locations of these peaks are contracted toward the nucleus due to effect of nuclear charge increases.

Table 2: The values of positions and maximum values of the $D\left(r_{1}\right)$ for studied system for $2 S$ shell.

\begin{tabular}{|c|c|c|c|c|}
\hline \multirow{2}{*}{ Atom } & \multicolumn{4}{|c|}{ 2S-shell } \\
\cline { 2 - 5 } & \multicolumn{2}{|c|}{ Peak 1 } & \multicolumn{2}{c|}{ Peak 2 } \\
\cline { 2 - 5 } & $\mathrm{R}_{1}$ & $\mathrm{D}_{\max }\left(\mathrm{r}_{1}\right)$ & $\mathrm{R}_{1}$ & $\mathrm{D}_{\max }\left(\mathrm{r}_{1}\right)$ \\
\hline $\mathrm{Be}$ & 0.21 & 0.053 & 2.10 & 0.396 \\
\hline $\mathrm{B}$ & 0.18 & 0.082 & 1.55 & 0.533 \\
\hline $\mathrm{C}$ & 0.15 & 0.111 & 1.20 & 0.663 \\
\hline $\mathrm{N}$ & 0.12 & 0.139 & 1.00 & 0.791 \\
\hline
\end{tabular}

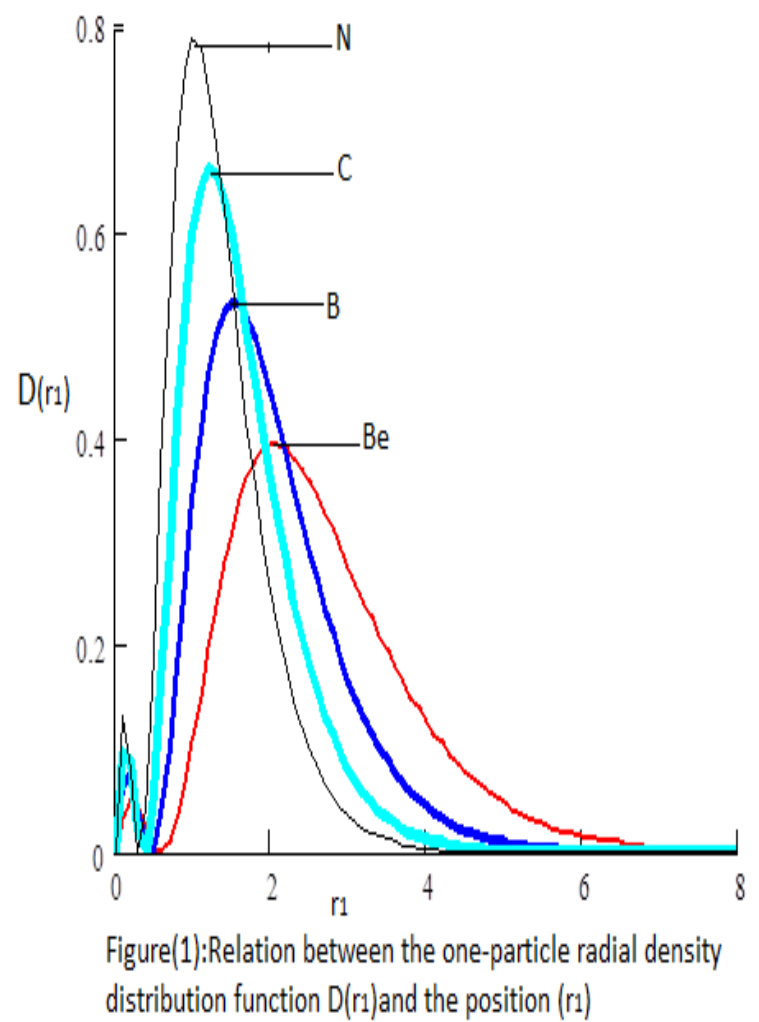

\section{One-particle expectation value $\left\langle r^{m}{ }_{1}\right\rangle$}

The one-particle expectation value and the standard deviation $\Delta r_{1}$ were calculated by using the following equations [5]:

$$
\begin{aligned}
& \left\langle r_{1}^{m}\right\rangle=\int_{0}^{\infty} D\left(r_{1}\right) r_{1}^{m} d r_{1} \\
& \Delta r_{1}=\left[\left\langle r_{1}^{2}\right\rangle-\left\langle r_{1}\right\rangle^{2}\right]^{1 / 2}
\end{aligned}
$$

Consecutively for different values for $\mathrm{m}$ where $\mathrm{m}$ is integer number and takes the values $-2 \leq \mathrm{m} \leq 2$, the results of the radial expectation values of $\left\langle\mathrm{r}_{1}{ }^{\mathrm{m}}\right\rangle$ and the standard deviation for $2 \mathrm{~S}$ shell of studied atoms are tabulated in Table 3. 
Table 3:The values of one-particle expectation values and standard deviation for $2 S$ shell of studies atoms.

\begin{tabular}{|c|c|c|c|c|c|c|c|}
\hline \multicolumn{2}{|c|}{} & & \multicolumn{2}{c|}{} & & \\
\multicolumn{2}{|c|}{ Atom } & $\left\langle r_{1}^{-2}\right\rangle$ & $\left\langle r_{1}^{-1}\right\rangle$ & $\left\langle r_{1}^{0}\right\rangle$ & $\left\langle r_{1}^{1}\right\rangle$ & $\left\langle r_{1}^{2}\right\rangle$ & $\Delta r_{1}$ \\
\hline \multirow{2}{*}{ Be } & Present work & 1.056119 & 0.522515 & 0.9999993 & 2.649405 & 8.426407 & 1.18619 \\
\cline { 2 - 8 } & Ref.[6] & 1.055647 & 0.522522 & -- & 2.649414 & 8.426431 & - \\
\hline \multirow{2}{*}{ B } & Present work & 2.04951 & 0.71887 & 1.0000011 & 1.977096 & 4.709244 & 0.89461 \\
\cline { 2 - 8 } & Ref.[6] & 2.024533 & 0.712882 & - & 1.977064 & 4.709135 & - \\
\hline \multirow{2}{*}{$\mathrm{C}$} & Present work & 3.255826 & 0.896783 & 1.000008 & 1.589342 & 3.05171 & 0.72504 \\
\cline { 2 - 8 } & Ref.[6] & 3.255510 & 0.896798 & - & 1.589344 & 3.052064 & - \\
\hline \multirow{2}{*}{$\mathrm{N}$} & Present work & 4.754181 & 1.078189 & 1.000011 & 1.332295 & 2.149463 & 0.61193 \\
\cline { 2 - 8 } & Ref.[6] & 4.753636 & 1.078176 & - & 1.332277 & 2.149438 & - \\
\hline
\end{tabular}

From Table 3 we noted :

The one-particle expectation value $\left\langle\mathrm{r}_{1}{ }^{\mathrm{m}}\right\rangle$ increase as the atomic number $(\mathrm{Z})$ increases for negative value of $\mathrm{m}$, The $\left\langle r_{1}^{-1}\right\rangle$ represents to the attraction energy expectation value and $\left\langle r_{1}^{-2}\right\rangle$ indicates how the density distribution in near regions of the nucleus.

For positive value of $m$ the one particle expectation value decreases as $\mathrm{Z}$ increases.

The standard deviation for all studied systems $\Delta r_{1} \quad$ decreases with increasing atomic number, because the distance between electron-nucleus becomes small.

Inter-particle radial density distribution function $f\left(r_{12}\right)$

The inter-particle radial density distribution function $f\left(r_{12}\right)$ was evaluated using the following equation below [7]:

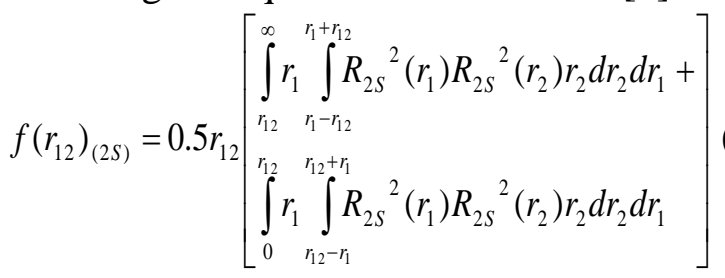

where $f\left(r_{12}\right)$ represented the Coulomb repulsion between a pair of electron in Lshell. The results for all systems as tabled in Table 4, and Fig. 2 indicates the relation between $f\left(r_{12}\right)$ and inters electronic distance $\mathrm{r}_{12}$.

Table4:The location and maximum values of $f\left(r_{12}\right)$ for studied systems.

\begin{tabular}{|c|c|c|}
\hline Atom & $r_{12}$ & $f\left(r_{12}\right)$ \\
\hline $\mathrm{Be}$ & 3.33 & 0.251 \\
\hline $\mathrm{B}$ & 2.5 & 0.336 \\
\hline $\mathrm{C}$ & 2 & 0.417 \\
\hline $\mathrm{N}$ & 1.67 & 0.497 \\
\hline
\end{tabular}




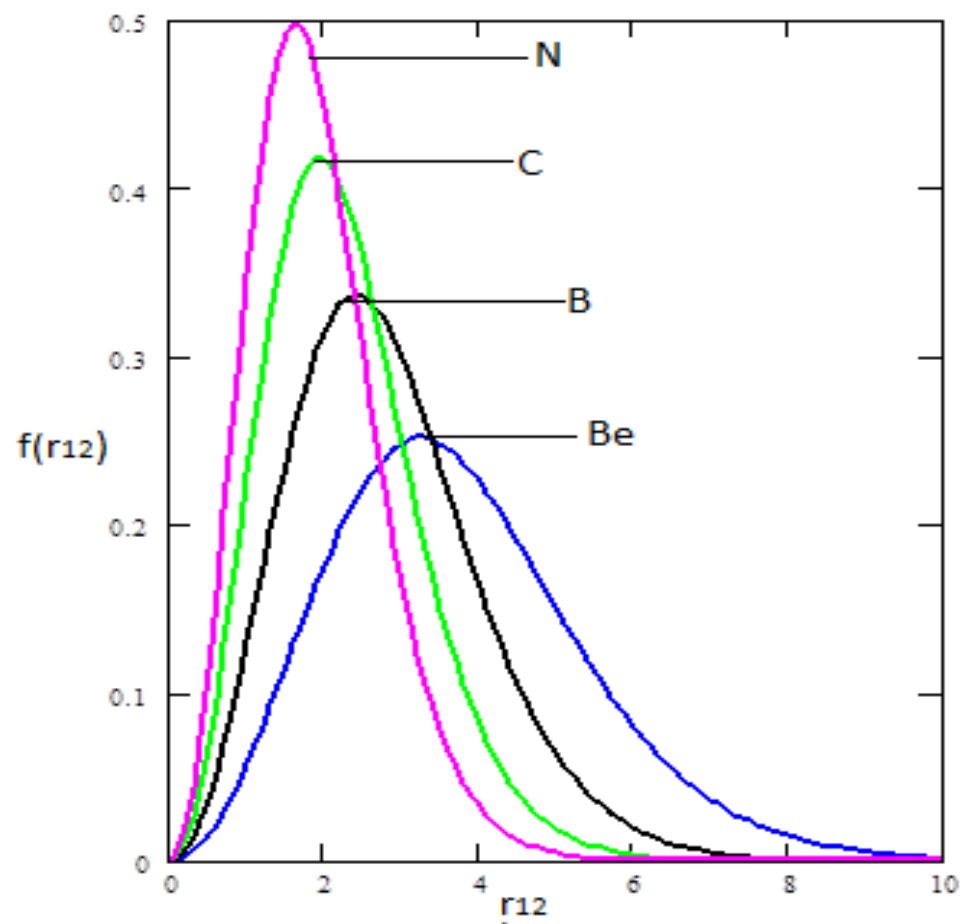

Figure(2):Relation between inter-particle radial density distribution function and the distance ( $\mathrm{r} 12)$.

From Table 4 and Fig. 2 we notes:

As atomic number increase, the distance $r_{12}$ between two electron decrease, this behavior from the fact that, each shell shrinkage toward the nucleus because the Coulomb attraction force will be greater than the Coulomb repulsion force between unlike spin in $2 \mathrm{~S}$ shell.

The maximum value $f\left(r_{12}\right)$ increases as $\mathrm{Z}$ increases because of increasing the atomic number which lead to increases the attraction force between the nucleus and the electrons.
Inter-particle expectation value $\left\langle r_{12}^{m}\right\rangle$

The inter-particle expectation value $\left\langle\boldsymbol{r}_{12}^{m}\right\rangle$ and standard deviation $\Delta r_{12}$ can be defined as [8]:

$$
\begin{aligned}
& <r_{12}{ }^{m}>=\int_{0}^{\infty} f\left(r_{12}\right) r_{12}{ }^{m} d r_{12} \\
& \Delta r_{12}=\sqrt{<r_{12}{ }^{2}>-<r_{12}>^{2}}
\end{aligned}
$$


Table 5: expectation values $\left\langle r_{12}^{m}\right\rangle$ where $-2 \leq m \leq 2$ and standard deviation.

\begin{tabular}{|c|c|c|c|c|c|c|c|}
\hline \multicolumn{2}{|c|}{ Atom } & $\left\langle r_{12}^{-2}\right\rangle$ & $\left\langle r_{12}^{-1}\right\rangle$ & $\left\langle r_{12}^{0}\right\rangle$ & $\left\langle r_{12}^{1}\right\rangle$ & $\left\langle r_{12}^{2}\right\rangle$ & $\begin{array}{c}\Delta r_{12} \\
\text { Standard } \\
\text { deviation }\end{array}$ \\
\hline \multirow{2}{*}{ Be } & Present work & 0.19041 & 0.34322 & 0.9999 & 3.75523 & 16.8527 & 1.65859 \\
\cline { 2 - 8 } & Ref.. [ 9] & 0.19041 & 0.34322 & 1.00000 & 3.73524 & 16.85233 & 1.65846 \\
\hline \multirow{2}{*}{ B } & & 0.34456 & 0.46027 & 1.00002 & 2.80513 & 9.41859 & 1.24492 \\
\hline \multirow{2}{*}{ C } & & 0.53609 & 0.57288 & 1.00002 & 2.25673 & 6.10347 & 1.00531 \\
\hline \multirow{2}{*}{ N } & & 0.76614 & 0.68376 & 1.0002 & 1.89291 & 4.29894 & 0.84606 \\
\hline
\end{tabular}

from analyzing the results in Table 5 we noted :

The inter-particle expectation value $\left\langle r_{12}^{m}\right\rangle$ increase as $\mathrm{Z}$ increases for -ve values of $m$, where as these values decreases for positive of $\mathrm{m}$. The standard deviation decrease when $\mathrm{Z}$ increase for all systems. The standard deviation contains much information concerning the shape of the two-particle radial density distribution function $f\left(r_{12}\right)$, the $\Delta r_{12}$ shape results indicate $f\left(r_{12}\right)$ become less diffuse.

The expectation values of potential energy $\langle V\rangle$

The total energy and the potential energy in atomic unit can be written as [8].

$$
\begin{aligned}
& \langle V\rangle=-Z\left\langle\frac{1}{r_{1}}\right\rangle+\left\langle\frac{1}{r_{12}}\right\rangle \\
& \langle T\rangle=\frac{\langle V\rangle}{2}
\end{aligned}
$$

and the results are listed in Table 6.
Table 6: The expectation values for attraction, repulsion and kinetic energies of the $2 s$ shell for studied atoms.

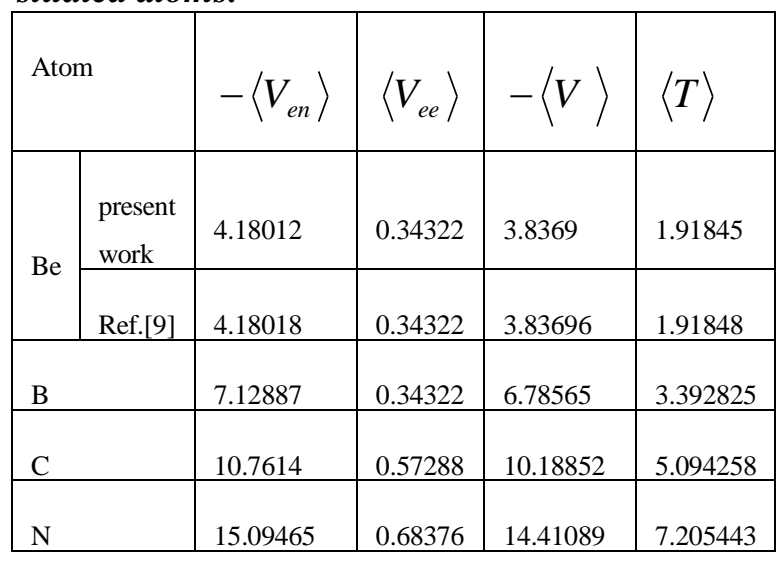

From the Table 6, The results of the expectation values for attraction potential $\left\langle V_{e n}\right\rangle$ and the repulsion potential $\left\langle V_{e e}\right\rangle$ nucleus-electron, and the results the expectation values of the total energy or Hartree-Fock energy are consistent with published results. Both $\left\langle V_{e n}\right\rangle$ and $\left\langle V_{e e}\right\rangle$ increase when atomic number increases. This result can be understood from the fact that all shell shrink toward the nucleus due to the attraction force between the protons and the 
electrons increase because the distance between nucleus-electron and electronelectron decrease, this result leads to increase in repulsion potential energy and attraction potential energy, the expectation values for total potential energy $\langle V\rangle$ increase in each system because the increase of $\left\langle V_{e n}\right\rangle$ are larger than that of $\left\langle V_{e e}\right\rangle$. we noted that the increase the energy of attraction is greater that the increase in the energy of repulsion when $\mathrm{Z}$ increase in order to maintain the entity of $v$ the atom. The expectation energy for kinetic energy $\langle T\rangle$ increase when $\mathrm{Z}$ increases.

\section{Conclusions}

The kinetic and potential energies of selected atoms (Be, B,C AND N) have been investigated theoretically. It was found that these energies increased with respect to atomic numbers $\mathrm{Z}$. Anther atomic properties such as one-particle radial density distribution function $D\left(r_{1}\right)$ and inter-particle radial distribution function $f\left(r_{12}\right)$ were studied. the results showed $D\left(r_{1}\right)$ and $f\left(r_{12}\right)$ were increased with atomic number. Moreover, the expectation values of position $\left\langle r^{m}{ }_{1}\right\rangle$ was in perfect agreement with published results for various values of $\mathrm{m}(-2,-1,1$ and 2$)$.
[1]E.F.Al-Kunani, "Theoretical Study of Radial Correlation and Other Atomic Properties for He Atom and He- like ions" M.Sc.,Thesis, College of Science, Nahrain University, (2007).

[2] C.Argaez, M.Melgaard, J.Nonlinear Analysis, 75 (2012) $384-404$.

[3] E. P. Lopez "Physical Chemistry: A practical approach", P.99, Williamstown, MA 01267 (1995).

[4] E.Clementi and Rotti, Atomic Data and Nuclear Data Tables, 14, 3-4 (1974) 177478.

[5] C.Chen, J. the European Physical D, 56 (2010) 303-309.

[6] Atomic Data and Nuclear Data Table, 95 (2009) 836-870.

[7]P.G.Giorgi and A.Svin, J.Physical Review, A.V.71, 032513(2005) 1-9.

[8] B.H.Asaad, "A study of the physical properties for the electrons outer shells for some atoms" M.Sc., Thesis, College of Science for Women Baghdad University (2005).

[9] K.S. Al-Khafiji, S.A. Al-Shebly, S.Farhan, Journal of Kufa-Physics, Special Issue, 20775830 (2010) 587-597.

\section{References}

